\title{
Retrospective Seroepidemiology study of dengue virus infection in Taiwan
}

\author{
Ying-Hsuan Lee ${ }^{1}$, Yu-Chia Hsieh ${ }^{1,2}$, Chih-Jung Chen ${ }^{1,2}$, Tzou-Yien Lin ${ }^{1,2}$ and Yhu-Chering Huang ${ }^{1,2^{*}}$
}

\begin{abstract}
Background: Dengue virus infection has been an important and serious public health concern in Taiwan, where local outbreaks of dengue fever occurred almost every year. To our knowledge, no nationwide investigation has been carried out to determine the actual extent of infection in the general population.

Methods: A total of 1308 random serum samples were collected from the general population in Taiwan in 2010. The antibody-captured enzyme-linked immunosorbent assays were used to detect DENV-specific IgM and IgG. Demographics data were used for risk analysis.

Results: The weighted overall seroprevalence was 1.96\% for anti-DENV IgM, and 3.4\% for anti-DENV IgG, respectively. A significant rise of DENV IgG seropositive rate had been noted since late adulthood stage, from 1.1\% at the age group of 50-59 years to $7.6 \%$ at the age group of 60-69 years. For people aged over 70 years, the seropositive rate reached 19\%. Age, nationality, and regions of residency were associated with the lgG seropositivity. There was no statistically significant difference in seroprevalence of anti-Dengue lgM, indicating recent infection, among univariate predictors we proposed, including gender, age, residency, nationality, and household size.

Conclusions: Our results indicated that the majority of population in Taiwan born after 1940 is naive to dengue virus and the prevalence of IgG antibody against dengue virus rises with age. Nationality, and regions of residency are associated with the exposure of population to infection by dengue viruses. Further studies are needed to realize the current situation of seroprevalence of dengue fever in Taiwan.
\end{abstract}

Keywords: Seroepidemiology, Dengue fever, Taiwan, Nationality

\section{Introduction}

Dengue infection is a major global burden caused by four types of dengue viruses (DENV-1, DENV-2, DENV3, DENV-4) belonging to Flaviviridae family. The viruses are transmitted through the bite of infected Aedes aegypti and Aedes albopictus female mosquitoes. Recovery from one episode of infection provides lifelong immunity against that particular serotype, yet the crossimmunity to the other serotypes is temporary and partial

\footnotetext{
* Correspondence: ychuang@adm.cgmh.org.tw

${ }^{1}$ Chang Gung University College of Medicine, Gueishan, Taoyuan, Taiwan ${ }^{2}$ Department of Pediatrics, Division of Pediatric Infectious Diseases, Chang Gung Memorial Hospital, No. 5, Fu-Shin Street, Gueishan, 333 Taoyuan, Taiwan
}

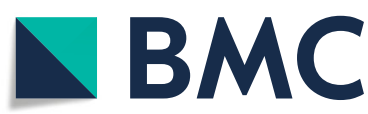

(c) The Author(s). 2021 Open Access This article is licensed under a Creative Commons Attribution 4.0 International License, which permits use, sharing, adaptation, distribution and reproduction in any medium or format, as long as you give appropriate credit to the original author(s) and the source, provide a link to the Creative Commons licence, and indicate if changes were made. The images or other third party material in this article are included in the article's Creative Commons licence, unless indicated otherwise in a credit line to the material. If material is not included in the article's Creative Commons licence and your intended use is not permitted by statutory regulation or exceeds the permitted use, you will need to obtain permission directly from the copyright holder. To view a copy of this licence, visit http://creativecommons.org/licenses/by/4.0/. The Creative Commons Public Domain Dedication waiver (http://creativecommons.org/publicdomain/zero/1.0/) applies to the data made available in this article, unless otherwise stated in a credit line to the data. infections.

Clinical manifestations of dengue virus infection range from asymptomatic, mild flu-like symptoms, to severe life-threatening dengue complications such as dengue shock syndrome (DSS) and dengue hemorrhagic fever (DHF) [3]. DHF/DSS cases are associated with a secondary-type dengue antibody response, which makes the second dengue infection worse than the first due to "antibody-dependent enhancement of infection" [3]. Those asymptomatic infection cases, which induce an antibody response but lacks clinical symptoms requiring a medical consultation, pose challenges to disease prevention programs. 
The incidence of dengue has grown dramatically worldwide in recent years. Recent studies estimated that 500, 000 people with severe dengue require hospitalization each year, and about $2.5 \%$ of those affected die $[4,5]$. Besides the growing incidence globally, the hyper-endemicity of multiple dengue virus serotypes in many regions also pose an alarming impact on public heath as well as national economies.

According to the previous study, Taiwan is not yet a dengue endemic region. Local outbreaks of dengue fever, however, occurred almost every year, which is believed to result from the constant importation of dengue infections from neighboring Southeast Asian countries [6]. The majority of outbreaks were reported in southern Taiwan, but occasionally in northern Taiwan [1, 7]. Notably, two consecutive severe dengue epidemics occurred in 2014 and 2015 in southern Taiwan [8]. The death rate in 2015 DHF/DSS cases was higher than previous dengue epidemics or outbreaks.

In recent years, arboviruses that cause dengue, chikungunya, and Zika illnesses have rapidly expanded across the globe. The more recent Zika outbreaks have caused severe neurological complications including GuillainBarré Syndrome and birth defects, of which the immunopathogenesis is enhanced in the setting of high seroprevalence of dengue antibodies due to structural similarities between Zika and dengue virus [9, 10]. It is important to study dengue virus seroprevalence to project future epidemic patterns in Taiwan. There have been scanty studies of seroprevalence of dengue virus from Taiwan, all from the southern part of Taiwan but not the whole island, and few results were published in the literature. Considering the massive population morbidity and the high rates of asymptomatic cases, existing studies were not enough for determining the current immune status of dengue in the community [11-13]. This pilot study was the first study to investigate the seroprevalence, measured by the presence of IgG and IgM antibodies of dengue virus in general populations from different geographical areas in Taiwan to figure out the whole picture of dengue disease prevalence in Taiwan.

\section{Materials and methods}

\section{Study samples}

In this study, 1308 human sera were selected from samples collected for one survey in 2010 [14]. This survey was conducted to investigate the seroprevalence of the pandemic influenza A H1N1 virus in Taiwan and a total of 1558 samples were obtained between September and October of 2010. Briefly, the survey used a multistratified design to sample the civilians from three regions (northern part of Taiwan: Taipei, Taoyuan; and southern part of Taiwan: Tainan). In each region, ageand gender-stratified sampling was conducted using household registration records. A questionnaire-based interview was used to collect demographic data at the time of blood sampling. Information about travel history (history of traveling to an endemic area), movement history, types of dwelling. Etc., was not collected in the serosurvey in 2010 and therefore not analyzed in the study.

To examine the DENV seroprevalence in the Taiwanese population, we selected residual samples from all age groups. Age was grouped into eight categories: 0-9, 10$19,20-29,30-39,40-49,50-59,60-69$, and $\geqq 70$ years. The serum samples were stored at $-80^{\circ} \mathrm{C}$.

\section{Ethical review}

The Institute Review Board of Chang Gung Memorial Hospital approved this study and all subjects provided written informed consent.

\section{Laboratory methods}

The DENV-specific IgM and IgG of the samples were determined by 2 commercial antibody-captured enzyme - linked immunosorbent assay (ELISA) systems (EUROIMMUN, Germany). The sensitivity and specificity of the IgM ELISA kit were 100 and 98\%, respectively, with a cut-off ratio of 1.0 relative units $(\mathrm{RU}) / \mathrm{ml}$. As for the IgG ELISA kit, the test showed a specificity of $100 \%$ and a sensitivity of $100 \%$, with a cut-off ratio of 1.1 relative units $(\mathrm{RU}) / \mathrm{ml}$. In addition, both the intra- and interassay coefficients of variability were less than $10 \%$ for anti-DENV IgM and anti-DENV IgG kits, respectively.

The analysis of anti-DENV responses was performed following the manufacturer's instructions. In short, the serum was diluted 1:100 and loaded to the pre-coated microplates. After incubation at room temperature for $30 \mathrm{~min}$, the plates were washed and incubated with peroxidase-labelled anti-human IgM/ IgG at room temperature for $30 \mathrm{~min}$. Following the further wash, the plates were incubated with kit substrates at room temperature for $15 \mathrm{~min}$. Positive controls, negative controls, and calibration standards were included on each plate. Photometric measurement of the color intensity was made at a wavelength of $450 \mathrm{~nm}$. The standard curve was established by calibrators and used to calculate the antibody concentration.

According to the previous reviews, antibodies of class IgM are detectable from the 5th day of illness and for 36 months following initial infection $[15,16]$. IgM antibodies are often not detectable after a second infection with another serotype [17]. Antibodies of class IgG arise several days later than IgM and probably persist for life. Therefore, individuals found positive for IgG were classified as those with past dengue virus infection, while those found positive for IgM were classified as recent dengue virus infection. 


\section{Statistics}

The data were analyzed with the SPSS Statistics, version 26.0 (2019 SPSS Inc., Chicago, IL, USA). In the interest of representativity as regard to the general population, each participant was weighted by post stratification according to age, gender in order to account for unequal probabilities of selection. In weighting, the structure of the total population in Taipei, Taoyuan, and Tainan from 2010 census data was used as the reference (available at https://www. ris.gov.tw/346). Weighted seroprevalence data are reported as percentages (\%) with a 95\% confidence interval. Comparison of population profile in general population and subjects recruited in this study was illustrated in Table 1. Regarding missing data for certain categories, percentages were determined by excluding those with missing data from the denominator.

Individual univariate qualitative variables were first analyzed for association with dengue seropositivity. Chisquared test was used to test for statistical significance in categorical variables as appropriate. Spearman's correlation coefficient was used to examine the strength of relationship between the age and the seropositive rate. An analysis of variance (ANOVA) used for comparisons of IgM/ IgG between cities. Variables with a $P$ value less than 0.20 were entered into the multivariable logistic regression model and odds ratios along with $95 \% \mathrm{CI}$ were reported [18]. All $P$ values reported were two- sided and statistical significance was taken at $P<0.05$.

\section{Results}

Demographic profile of participants

The demographic characteristics of the participants are shown in Table 1. The 1308 serum samples were obtained from 791 (60.5\%) females and 517 (39.5\%) males. The mean age of the participants was 42.4 years (range 0-92 years, standard deviation 21.4). Among them, 983 subjects (75.2\%) and 325 (24.8\%) were from northern district (Taipei and Taoyuan) and southern district (Tainan), respectively.

\section{Seropositivity of anti-DENV IgM among participants}

Of the 1308 study subjects, the overall weighted seropositive rate of anti-DENV IgM was $1.76 \%$ (95\%CI, 1.74-1.78, Table 2) Weighted seropositive rates among females and males were $2.47 \%$ (95\% CI, 2.42-2.52\%) and $1.21 \%$ (95\% CI, 1.18-1.24\%), respectively. The incidence of IgM antibodies was highest in the age group of 0-9 years $(3.70,95 \% \mathrm{CI}, 3.39-4.01 \%)$ followed by the age group of those 70 years or above $(3.47,95 \%$ CI, 3.08$3.81 \%)$; seroprevalence fluctuated between $0.61 \sim 2.13 \%$ for participants aged $10 \sim 69$ years. No statistical relationship was observed between IgM-positive subjects and age groups $(p=0.658)$.

Participants in Taoyuan had the highest weighted seroprevalence of anti-DENV IgM $(2.18,95 \%$ CI, 2.12 $2.25 \%)$, followed by Taipei $(1.75,95 \%$ CI, $1.70-1.80 \%)$ and Tainan (1.50, 95\% CI, 1.43-1.57\%). DENV IgM was not detected in any of the sera samples collected from

Table 1 Comparison of population profile in Taiwan and subjects recruited in the study

\begin{tabular}{|c|c|c|c|c|}
\hline \multirow[t]{2}{*}{ Characteristics } & \multicolumn{2}{|c|}{ Subjects recruited in this study } & \multicolumn{2}{|c|}{${ }^{\mathrm{a}} 2010$ population by census } \\
\hline & Number & $\%$ & Number & $\%$ \\
\hline \multicolumn{5}{|l|}{ Gender } \\
\hline Male & 517 & 39.5 & $5,151,540$ & 49.6 \\
\hline Female & 791 & 60.5 & $5,240,453$ & 50.4 \\
\hline \multicolumn{5}{|c|}{ Age groups (years) } \\
\hline $0-9$ & 246 & 18.8 & 944,019 & 9.1 \\
\hline $10-19$ & 154 & 11.8 & $1,371,595$ & 13.2 \\
\hline $20-29$ & 180 & 13.8 & $1,542,079$ & 14.8 \\
\hline $30-39$ & 196 & 15 & $1,747,486$ & 16.8 \\
\hline $40-49$ & 178 & 13.6 & $1,728,634$ & 16.6 \\
\hline $50-59$ & 177 & 13.5 & $1,555,250$ & 15.0 \\
\hline $60-69$ & 119 & 9.1 & 780,058 & 7.5 \\
\hline$\geq 70$ & 58 & 4.4 & 722,872 & 7.0 \\
\hline \multicolumn{5}{|l|}{ Locality } \\
\hline Taipei & 494 & 37.8 & $6,516,139$ & 62.7 \\
\hline Taoyuan & 489 & 37.4 & $2,002,060$ & 19.3 \\
\hline Tainan & 325 & 24.8 & $1,873,794$ & 18.0 \\
\hline
\end{tabular}

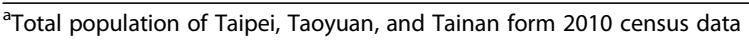


Table 2 Univariate predictors of dengue lgM seropositivity in Taiwan

\begin{tabular}{|c|c|c|c|c|c|c|}
\hline Characteristics & No. positive/ No. tested & Weighted prevalence (\%) & $(95 \% \mathrm{Cl})$ & Odds Ratio & $95 \% \mathrm{Cl}$ & ${ }^{*} P$ value \\
\hline Gender & & & & & & 0.009 \\
\hline Male & $10 / 517$ & 1.21 & & Referent & & \\
\hline Female & 18/791 & 2.47 & & 2.071 & $(0.877,4.889)$ & \\
\hline Age groups (years) & & & & & & 0.658 \\
\hline $0-9$ & $10 / 246$ & 3.70 & $(3.39,4.01)$ & Referent & & \\
\hline $10-19$ & 0/154 & 0.61 & $(0.52,0.69)$ & 0.159 & $(0.019,1.356)$ & \\
\hline $20-29$ & $2 / 180$ & 1.36 & $(1.25,1.48)$ & 0.36 & $(0.077,1.683)$ & \\
\hline $30-39$ & $4 / 196$ & 1.57 & $(1.46,1.68)$ & 0.414 & $(0.099,1.728)$ & \\
\hline $40-49$ & $5 / 178$ & 1.56 & $(1.44,1.67)$ & 0.411 & $(0.098,1.728)$ & \\
\hline $50-59$ & $3 / 177$ & 2.13 & $(1.98,2.27)$ & 0.566 & $(0.145,2.410)$ & \\
\hline $60-69$ & $2 / 119$ & 1.92 & $(1.65,2.20)$ & 0.51 & $(0.091,2.870)$ & \\
\hline$\geq 70$ & $2 / 58$ & 3.47 & $(3.08,3.87)$ & 0.936 & $(0.215,4.081)$ & \\
\hline Total & & 1.76 & $(1.74,1.78)$ & & & \\
\hline Locality & & & & & & 0.766 \\
\hline Taipei & 9/ 494 & 1.75 & & Referent & & \\
\hline Taoyuan & $12 / 489$ & 2.18 & & 1.252 & $(0.506,3.097)$ & \\
\hline Tainan & $7 / 325$ & 1.50 & & 0.855 & $(0.282,2.590)$ & \\
\hline Nationality & & & & & & 0.582 \\
\hline Taiwanese & $27 / 1227$ & 1.83 & & & & \\
\hline Immigrants & $0 / 21$ & 0 & & & & \\
\hline Household size & & & & & & 0.455 \\
\hline $1-4$ & $13 / 648$ & 1.66 & & Referent & & \\
\hline$\geq 5$ & $10 / 456$ & 2.28 & & 1.402 & $(0.591,3.33)$ & \\
\hline
\end{tabular}

$\mathrm{Cl}$ Confidence interval. Prevalence, Odds Ratio, and Cls are based on weighted data

Missing data: Nationality $(n=60)$, Household size $(n=205)$. Percentages determined by excluding those with missing data from the denominator

One sample collected from one 51-year-old male, who lived in Taipei with a household size of four, showed positive for both IgM and IgG

immigrants, while the seroprevalence of DENV IgM in Taiwanese participants was $1.83 \%$.

Based on univariate analysis, the relationship between DENV IgM and the other sociodemographic characteristics (age, region of residence, nationality, or household size) did not show any statistically significant association (Table 2).

\section{Seropositivity of anti-DENV IgG among participants}

The overall weighted seropositive rate of anti-DENV IgG was $3.40 \%$ (95\% CI, 3.37-3.42\%). The weighted seropositive rates among women and men were $3.52 \%(95 \% \mathrm{CI}$, $3.47-3.58 \%$ ), and $3.27 \%(95 \% \mathrm{CI}, 3.21-3.32 \%$ ) respectively. No significant difference in seropositivity of IgG between male and female participants was detected ( $p=$ 0.796).

According to the univariate analysis, statistically significant differences in seropositivity of IgG were found between age group $(p<0.0001)$ (Table 3). A significant rise of DENV IgG seropositive rate had been noted among those ages 50 and over, from $1.23 \%$ at the age group of $50-59$ years to $8.33 \%$ at the age group of $60-$
69 years. For people aged 70 years and above, the seropositive rate reached $18.25 \%$. In addition, the nationality (IgG seropositive rate among immigrants: $28.82 \%$, $p<0.0001$ ) was associated with the DENV IgG seropositivity. Region of residence was statistically associated with seropositive rates of DENV IgG $(p=0.001)$; the prevalence of dengue IgG positives were highest in subjects residing in Tainan with $6.64 \%$, followed by $2.76 \%$ in Taipei and $1.81 \%$ in Taoyuan. No statistical relationship was observed between IgG-positive subjects and household size $(p=0.302)$.

In the multivariate logistic regression model, individuals aged 60 or older, immigrant and individuals living in Tainan were found to be independent risk factors significantly associated with IgG seroposivity (Table 4).

\section{The joint seropositivity of antibodies (Ab) IgM and IgG} The entire weighted seroprevalence of dengue-specific antibodies (IgM and/or IgG) in the population studied was $6.78 \%$. Among the study subjects, only one sample showed seropositive to both IgM and IgG. 
Table 3 Univariate analysis of dengue lgG seropositivity in Taiwan

\begin{tabular}{|c|c|c|c|c|c|c|}
\hline Characteristics & No. positive/ No. tested & Weighted prevalence (\%) & & Odds Ratio & $95 \% \mathrm{Cl}$ & ${ }^{*} P$ value \\
\hline Gender & & & & & & 0.796 \\
\hline Male & $18 / 517$ & 3.27 & & Referent & & \\
\hline Female & $266 / 791$ & 3.52 & & 1.082 & $(0.594,1.971)$ & \\
\hline Age groups (years) & & & & & & $<0.0001$ \\
\hline $0-9$ & $6 / 246$ & 2.41 & $(2.15,2.67)$ & Referent & & \\
\hline $10-19$ & $2 / 154$ & 1.21 & $(1.09,1.33)$ & 0.498 & $(0.082,3.004)$ & \\
\hline $20-29$ & $3 / 180$ & 1.62 & $(1.49,1.74)$ & 0.666 & $(0.132,3.36)$ & \\
\hline $30-39$ & 9/196 & 3.38 & $(3.23,3.54)$ & 1.419 & $(0.356,5.652)$ & \\
\hline $40-49$ & $2 / 178$ & 0.78 & $(0.69,0.86)$ & 0.318 & $(0.047,2.154)$ & \\
\hline $50-59$ & $2 / 177$ & 1.23 & $(0.13,1.33)$ & 0.504 & $(0.089,2.842)$ & \\
\hline $60-69$ & $9 / 119$ & 8.33 & $(7.79,8.88)$ & 3.684 & $(0.932,14.555)$ & \\
\hline$\geq 70$ & $11 / 58$ & 18.25 & $(17.41,19.09)$ & 9.046 & $(2.496,32.793)$ & \\
\hline Total & & 3.40 & $(2.30,4.49)$ & & & \\
\hline Locality & & & & & & 0.001 \\
\hline Taipei & $15 / 494$ & 2.76 & & Referent & & \\
\hline Taoyuan & $11 / 489$ & 1.81 & & 0.651 & $(0.275,1.537)$ & \\
\hline Tainan & $18 / 325$ & 6.64 & & 2.504 & $(1.262,4.969)$ & \\
\hline Nationality & & & & & & $<0.0001$ \\
\hline Taiwanese & $36 / 1227$ & 3.06 & & Referent & & \\
\hline Immigrants & $6 / 21$ & 28.82 & & 12.821 & $(4.180,39.325)$ & \\
\hline Household size & & & & & & 0.302 \\
\hline $1-4$ & $17 / 648$ & 2.67 & & Referent & & \\
\hline$\geq 5$ & $16 / 455$ & 3.76 & & 1.424 & $(0.725,2.795)$ & \\
\hline
\end{tabular}

Cl Confidence interval. Prevalence, Odds Ratio, and Cls are based on weighted data

Missing data: Nationality $(n=60)$, Household size $(n=205)$. Percentages determined by excluding those with missing data from the denominator

One sample collected from one 51-year-old male, who lived in Taipei with a household size of four, showed positive for both IgM and IgG

\section{Comparison of seroprevalence between regions}

The seroprevalence of antibodies against dengue virus for each region, by age and gender, is summarized ( $\mathrm{Ta}$ bles 5 and 6). As previously mentioned above, the overall seroprevalence of anti-DENV IgM was highest in Taoyuan (2.18, 95\% CI, 2.12-2.25\%), followed by Taipei (1.75, 95\% CI, 1.70-1.80\%) and Tainan (1.50, 95\% CI, $1.43-1.57 \%)$. Among individuals $0-9$ years of age, the highest prevalence was in Tainan (4.73\%). The prevalence of anti-dengue IgM was then declined and remained relatively low among individuals 10-69 years of age regardless of the region in which they resided $(0 \% \sim 2.78 \%)$ (Fig. 1). The seroprevalence sharply increased for individual aged 70 years and above in Taipei and Taoyuan, reaching 13.84 and $5.90 \%$, respectively. The shape of the curve of seropositivity by age between Taipei and Taoyuan was very similar. In Tainan, the seroprevalence was highest among individuals 0-9 years of age (4.73\%). No IgM seropositive case was detected in the 10-19-year-old, 50-year-old and over age group in Tainan.
Figure 2 shows the prevalence of anti-dengue IgG in different age groups by regions. There was a significant association for increasing DENV IgG seropositivity with increasing age, similar to patterns in the overall group. A sharp rise in DENV IgG seropositivity in people 60 years and older was observed. Among those over 60 years of age, the highest seroprevalence was found in Tainan (41.31\%), followed by that in Taipei $(34.16 \%)$ and in Taoyuan (5.90\%). As mentioned previously, there was a significant difference in the prevalence of DENV IgG among the 3 regions, and this difference was clearest in the age groups of 60 years and above.

\section{Discussion}

Of the overall weighted prevalence of dengue-specific IgM and IgG antibodies of $6.78 \%$, IgM was found positive in $1.76 \%$ and IgG in $3.40 \%$ of the study population. The seroprevalence of dengue antibodies in this study was lower than that reported from the epidemiological study conducted in nearby countries (39.3-59.0\%) including Bangladesh, Singapore and India [19-21], but 
Table 4 Multivariate logistic regression model of risk factors associated with dengue IgG seropositivity in Taiwan

\begin{tabular}{|c|c|c|c|c|}
\hline Characteristics & No. positive/ No. tested & Adjusted OR & $95 \% \mathrm{Cl}$ & $P$ value \\
\hline Nationality & & & & $<0.0001$ \\
\hline Taiwanese & $36 / 1227$ & Referent & & \\
\hline Immigrants & $6 / 21$ & 51.887 & $(12.766,210.898)$ & $<0.0001$ \\
\hline Age groups (years) & & & & $<0.0001$ \\
\hline $0-9$ & $6 / 246$ & (referent) & - & \\
\hline $10-19$ & $2 / 154$ & 0.574 & $(0.084,3.919)$ & 0.571 \\
\hline $20-29$ & $3 / 180$ & 0.840 & $(0.148,4.754)$ & 0.844 \\
\hline $30-39$ & 9/196 & 1.452 & $(0.315,6.698)$ & 0.632 \\
\hline $40-49$ & $2 / 178$ & 0.299 & $(0.039,2.294)$ & 0.246 \\
\hline $50-59$ & $2 / 177$ & 0.261 & $(0.022,3.118)$ & 0.289 \\
\hline $60-69$ & $9 / 119$ & 4.776 & $(1.045,21.839)$ & 0.044 \\
\hline$\geq 70$ & $11 / 58$ & 13.283 & $(3.144,56.112)$ & $<0.0001$ \\
\hline Locality & & & & $<0.001$ \\
\hline Taipei & $15 / 494$ & (referent) & & \\
\hline Taoyuan & $11 / 489$ & 0.526 & $(0.188,1.467)$ & 0.220 \\
\hline Tainan & $18 / 325$ & 3.262 & $(1.518,7.007)$ & 0.000 \\
\hline
\end{tabular}

$\mathrm{Cl}$ Confidence interval. Adjusted OR, and $\mathrm{Cls}$ are based on weighted data

Missing data: Nationality $(n=60)$. Percentages determined by excluding those with missing data from the denominator

Table 5 Seroprevalence of anti-dengue virus $\lg M$ and anti-dengue virus lgG stratified by age groups and cities $^{a}$

\begin{tabular}{|c|c|c|c|c|c|c|c|}
\hline \multicolumn{2}{|c|}{ Characteristics } & \multicolumn{2}{|l|}{ Taipei } & \multicolumn{2}{|l|}{ Taoyuan } & \multicolumn{2}{|l|}{ Tainan } \\
\hline & & Seroprevalence (\%) & $95 \% \mathrm{Cl}$ & Seroprevalence (\%) & $95 \% \mathrm{Cl}$ & Seroprevalence (\%) & $95 \% \mathrm{Cl}$ \\
\hline \multicolumn{8}{|c|}{ Age groups (years) } \\
\hline \multirow[t]{2}{*}{$0-9$} & $\lg M$ & 1.76 & $(1.29,2.22)$ & 3.12 & $(2.55,3.68)$ & 4.73 & $(3.45,6.01)$ \\
\hline & $\lg G$ & 0.93 & $(0.59,1.26)$ & 3.03 & $(2.76,3.30)$ & 1.58 & $(0.82,2.33)$ \\
\hline \multirow[t]{2}{*}{$10-19$} & $\lg M$ & 0.00 & $(0.00,0.00)$ & 1.50 & $(1.16,1.84)$ & 0.00 & $(0.00,0.00)$ \\
\hline & $\lg G$ & 0.00 & $(0.00,0.00)$ & 0.00 & $(0.00,0.00)$ & 4.48 & $(3.61,5.35)$ \\
\hline \multirow[t]{2}{*}{$20-29$} & $\lg M$ & 1.15 & $(0.88,1.42)$ & 1.40 & $(1.04,1.77)$ & 1.84 & $(1.29,2.39)$ \\
\hline & $\lg G$ & 0.00 & $(0.00,0.00)$ & 1.40 & $(1.02,1.79)$ & 4.69 & $(3.83,5.56)$ \\
\hline \multirow[t]{2}{*}{$30-39$} & $\lg M$ & 0.81 & $(0.64,0.99)$ & 2.59 & $(2.14,3.05)$ & 1.55 & $(1.10,2.01)$ \\
\hline & $\lg G$ & 4.07 & $(3.69,4.45)$ & 2.40 & $(1.92,2.87)$ & 3.11 & $(2.47,3.75)$ \\
\hline \multirow[t]{2}{*}{$40-49$} & $\lg M$ & 1.16 & $(0.87,1.45)$ & 1.01 & $(0.78,1.25)$ & 2.90 & $(2.34,3.47)$ \\
\hline & $\lg G$ & 1.16 & $(0.87,1.45)$ & 1.01 & $(0.75,1.27)$ & 0.00 & $(0.00,0.00)$ \\
\hline \multirow[t]{2}{*}{$50-59$} & $\lg M$ & 2.78 & $(2.41,3.16)$ & 2.64 & $(2.17,3.11)$ & 0.00 & $(0.00,0.00)$ \\
\hline & $\lg G$ & 2.78 & $(2.41,3.16)$ & 0.00 & $(0.00,0.00)$ & 0.00 & $(0.00,0.00)$ \\
\hline \multirow[t]{2}{*}{$60-69$} & $\lg M$ & 2.27 & $(1.75,2.80)$ & 1.89 & $(1.09,2.69)$ & 0.00 & $(0.00,0.00)$ \\
\hline & $\lg G$ & 3.41 & $(2.77,4.06)$ & 5.66 & $(4.59,6.74)$ & 18.92 & $(15.62,22.22)$ \\
\hline \multirow[t]{2}{*}{$\geq 70$} & $\lg M$ & 13.84 & $(8.43,19.25)$ & 5.90 & $(3.99,7.82)$ & 0.00 & $(0.00,0.00)$ \\
\hline & $\lg G$ & 34.1 & $(26.74,41.58)$ & 5.90 & $(2.84,8.97)$ & 41.31 & $(37.66,44.97)$ \\
\hline \multirow[t]{2}{*}{ Total } & $\lg M$ & 1.75 & $(1.70,1.80)$ & 2.18 & $(2.12,2.25)$ & 1.50 & $(1.43,1.57)$ \\
\hline & $\lg G$ & $2.76 \%$ & $(2.70,2.83)$ & $1.81 \%$ & $(1.77,1.86)$ & $6.64 \%$ & $(6.49,6.79)$ \\
\hline
\end{tabular}


Table 6 According to a database of dengue cases covering the period $1998 \sim 2010,8956$ of 14,013(63.9\%) confirmed dengue cases were reported from Kaohsiung

\begin{tabular}{ll}
\hline Region & Dengue Cases \\
\hline Taipei City & 298 \\
Taichung City & 175 \\
Tainan City & $\mathbf{2 8 5 3}$ (20.2\%) \\
Kaohsiung City & $\mathbf{8 9 5 6}$ (63.9\%) \\
Keelung City & 20 \\
Hsinchu City & 16 \\
Chiayi City & 13 \\
New Taipei City & 305 \\
Taoyuan City & 207 \\
Hsinchu County & 38 \\
Yilan County & 21 \\
Miaoli County & 29 \\
Changhua County & 79 \\
Nantou County & 31 \\
Yunlin County & 34 \\
Chiayi County & 33 \\
Pingtung County & 856 \\
Penghu County & 13 \\
Hualien County & 16 \\
Taitung County & 15 \\
Kinmen County & 4 \\
Lienchiang County & 1 \\
Nation-wide & 14,013 \\
\hline Based on informtion from the Center & \\
\hline
\end{tabular}

Based on information from the Center for Disease Control (CDC), Taiwan higher than that reported from Hong Kong (1.6\%) [22]. Previous reports indicated that Taiwan is not a dengue endemic region and that the constant importation of DENVs from neighboring Southeast Asian countries is the major cause of dengue outbreaks; this may explain the relatively low seroprevalence of dengue-specific antibodies compared with the nearby endemic countries [6]. The discrepancy between DENV seroprevalence in Taiwan and that in Hong Kong may result from species of vectors. Aedes aegypti, the primary species responsible for transmitting dengue viruses, was not found in Hong Kong after the mid-1950s [22], and Aedes albopictus alone contributes to the transmission of dengue fever.

It has been wildly accepted that dengue epidemics transmitted by Aedes albopictus tend to be mild and short-lived. Correspondingly, the distribution of dengue cases highly overlaps where Aedes aegypti but not Aedes albopictus breeds in the field according to the survey conducted by Taiwan Centers for Disease Control (CDC). However, recent studies proposed that the propagation efficiency of Aedes albopictus for DENV1 and DENV3 transmission was as high as that of Aedes aegypti and that their intimacy to people is the major reason why Aedes aegypti is the primary DENV vector. According to 2014 record-breaking dengue outbreak in Guangzhou and the ongoing locally transmitted dengue fever in Hong Kong, however, the role of Aedes albopictus in the transmission of dengue fever should not be neglected.

IgM- and IgG-capture ELISAs are widely used as diagnostic tests for confirmation of dengue virus infection and is considered to be a reliable serological test [23]. A patient who has IgM antibodies to dengue detected via

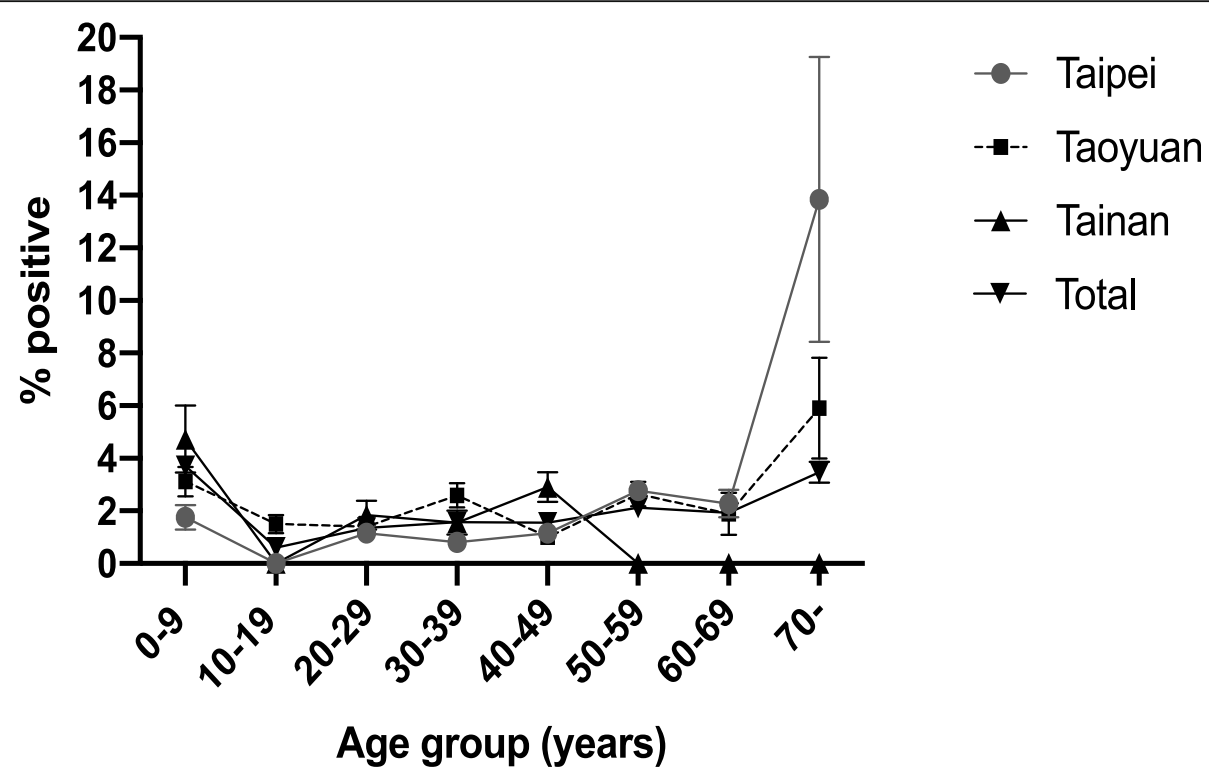

Fig. 1 Age group-specific anti-DENV IgM seroprevalence by region 


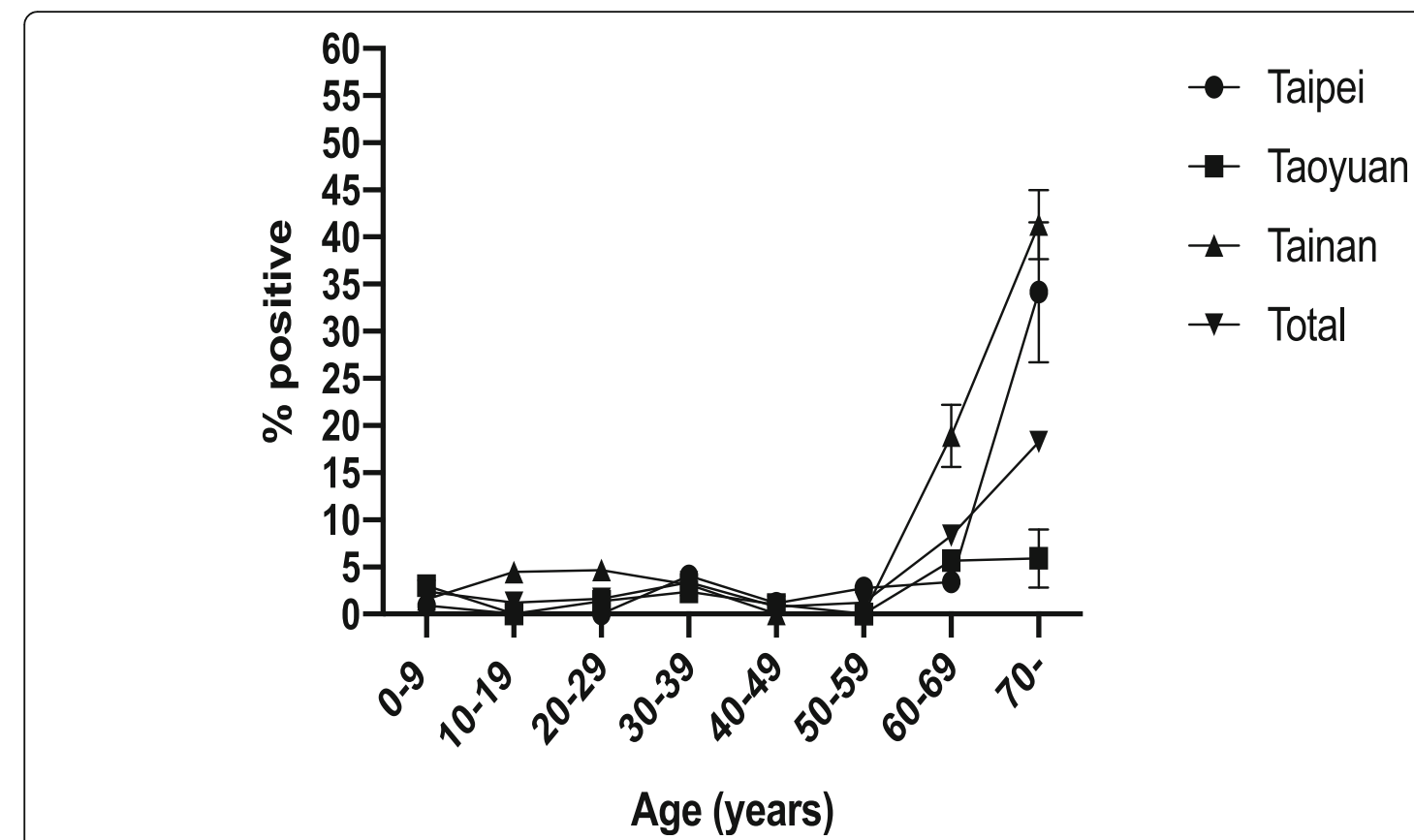

Fig. 2 Age group-specific anti-DENV IgG seroprevalence by region

ELISA indicates having a recent and primary dengue infection [24]. According to gender distribution, DENV IgM were more common in female subjects than males in this study, while no significant gender differences were observed for DENV IgG. These findings are comparable with the study done by Chien et al. [16].

In a serologic study of 1391 participants in in three administrative districts of Tainan in 2015, the seroprevalence of DENV IgM and DENV IgG were found to be increased with age [16]. In this study, however, there was no statistically significant difference in seroprevalence of DENV IgM among age groups, suggesting that age is not associated with the susceptibility to dengue fever. On the other hand, the multivariate analysis showed that older age was associated with DENV IgG seropositivity; most individuals less than 70 years old (born after 1940) were antibody negative (0-10\%), and the positive rate reached up to $22.41 \%$ for those over 70 years old. This increase in seroprevalence is likely a reflection of the increasing exposure as individuals' age. Moreover, the sharp increase in DENV seroprevalence in those aged 70 years and above could be partly attributed to the island-wide major outbreak of dengue in 1942, that resulted in 5 million infections among 6 million residents $(>80 \%)$ then [25]. The trends are in agreement with two other studies conducted in southern Taiwan (Tainan and Kaohsiung, respectively), in which drastic increase in seroprevalence of DENV IgG were found in both studies [16, 26, 27]. These findings also imply that the majority of population below 70 years of age are naive to all of the four dengue virus serotypes.
In the current study, region of residence was found to be significantly associated with the disparity of DENV IgG seropositivity. (Table 4) Individuals living in Tainan had 2.504 (95\% CI $=1.262,4.969)$ times the age-adjusted odds of being IgG seropositive compared with those living in Taipei, while those living in Taoyuan had 0.651 (95\% CI $=0.275,1.537)$ times the gender- and ageadjusted odds of being IgG seropositive compared with individuals living in Taipei. Considering the fact that the majority of outbreaks were reported from southern Taiwan, the discrepancy of seropositivity between Tainan and Taipei was relatively trivial. Population mixing facilitated by the efficiency of modern transportation networks may have accounted for that phenomenon. The relatively low dengue risk was observed in Taoyuan than that in Taipei may partially due to the lower population densities, lower people mixing with viremic individuals from southern Taiwan and lower people traveling abroad to dengue-endemic countries. Surprisingly, the seroprevalence of DENV IgMwas lowest in Tainan (1.50, 95\% CI, 1.43-1.57\%) and highest in Taoyuan (2.18, 95\% CI, 2.12-2.25\%), although the difference was not statistically significant. The similar probability of exposure may be explained by the fact that no dengue outbreak occurred in 2010. According to the reports from Taiwan Centers for Disease Control [28], a total of 1896 cases of dengue fever was reported in 2010 and 1106 cases resided in Kaohsiung City, accounting for $58.3 \%$ of the total cases. In the present study, the age -stratified seroprevalence of each city was investigated. As mentioned above, a sharp and significant 
increase in IgM seroprevalence from subjects aged below 70 years old to those $\geq 70$ y/o was observed in Taipei and Taoyuan. On the contrary, the IgM seroprevalence was highest among individuals $0-9$ years of age and no IgM seropositive case was detected in the 10-19-year-old, 50-year-old and over age group in Tainan. The absence of IgM antibodies in those age groups may be statistically error due to few dengue fever cases reported in 2010. Examining age patterns of DENV IgG seroprevalence, we found a sharp increase in DENV IgG seroprevalence for those aged 70 years and above presented in each city. These results confirmed the wide spread of dengue outbreak in 1942 or earlier. Moreover, the relatively low and stable rates among the age group of $<70$ years indicated that the impact of outbreaks after1942 were relatively limited.

According to the multivariate analysis, immigrants were at significantly increased risk of DENV IgG seroprevalence. Unfortunately, the original nationality was not collected in the serosurvey in 2010. Further investigation is required to determine whether these individuals have acquired dengue infections before coming to live in Taiwan. However, most of recent immigrants were due to marriage and were from mainland China and Southeastern Asian countries where dengue is endemic.

According to the current study, there was only one sample positive for both IgM and IgG. This sample was collected from one 51-year-old male, who lived in Taipei with a household size of four. Unfortunately, his nationality was not documented then.

There were several limitations in this seroprevalence study. Firstly, the delinked serum samples were collected prior to the 2014-2015 outbreak of dengue fever in southern Taiwan, our results may not be representative to the current population profile. Second, some epidemiological factors that may influence the seropositivity were not collected. Traveling history, for instance, may impose significant effect on the seroprevalence since many endemic areas in Southeast Asia are popular destinations for Taiwan citizens. Moreover, sera samples from Kaohsiung region (in the southern part of Taiwan) were not included in this study. There was almost 40 years of dormancy after the island-wide dengue outbreak in 1942. However, the reemergence of dengue fever was documented in 1981 and dengue fever outbreaks occurred of different scales then. According to a database covering the period from 1998 to 2010 based on information from the Taiwanese Center for Disease Control (CDC) (Table 6), Kaohsiung accounted for $63.9 \%$ of 14 , 013 dengue cases reported in the entire country, responsible for most of the dengue cases in Taiwan. Therefore, the overall dengue disease prevalence in Taiwan in this study may be underestimated to some extent. A more recent and the inclusion of more districts are required for more precise conclusions about the association between risk factors and dengue seroprevalence in the current general population of Taiwan.

Conclusively, this study provides the baseline seroprevalence data of dengue fever in Taiwan, which included subjects from northern district and southern district of Taiwan. Further studies, which should include more districts of Taiwan and more sera samples obtained after 2014-2015 outbreak of dengue in Taiwan, are needed to realize the current situation of seroprevalence of dengue fever in Taiwan.

\section{Abbreviations}

DENV: Dengue virus; DSS: Dengue shock syndrome; DHF: Dengue hemorrhagic fever; ELISA: Enzyme-linked immunosorbent assay

\section{Acknowledgements}

The authors thank the research assistant, Ms. Yi-Chen Kuan, for excellent technical assistance.

\section{Authors' contributions}

YHL: conception and design, acquisition of data, laboratory performance, analysis and interpretation of data, drafting the manuscript. YC Hsieh: conception and design, acquisition of data, analysis and interpretation of data. CJC: conception and design, acquisition of data, analysis and interpretation of data. TYL: conception and design, acquisition of data, analysis and interpretation of data. YC Huang: conception and design, analysis and interpretation of data, funding application, drafting the manuscript. All authors read and approved the final manuscript.

\section{Funding}

This study was supported by a grant from Chang Gung Memorial Hospital (CMRPG3H1291) and a grant from Medical Foundation in Memory of Dr. Deh-Lin Cheng. The funders had no role in the study design, data collection and analysis, decision to publish, or preparation of the manuscript.

Availability of data and materials

The datasets used and/or analysed during the current study are available from the corresponding author on reasonable request.

Ethics approval and consent to participate

This study was approved by the Institutional Review Board of Chang Gung Memorial Hospital. A written informed consent was obtained from the participants and participants' parents or guardians if needed.

Consent for publication

Not applicable.

\section{Competing interests}

The authors have no conflicts of interest relevant to this article to disclose.

Received: 17 March 2020 Accepted: 15 January 2021

Published online: 21 January 2021

\section{References}

1. Jason C. Trend and geographic analysis of the prevalence of dengue in Taiwan, 2010-2015. Intern J Infect Dis. 2017:54:43-9.

2. Kurane I, Matsutani T, Suzuki R, Takasaki T, Kalayanarooj S, Green S, et al. Tcell responses to dengue virus in humans. Trop Med Health. 2011;39(4 Suppl):45-51.

3. Pang $X$, Zhang $R$, Cheng $G$. Progress towards understanding the pathogenesis of dengue hemorrhagic fever. Virol Sin. 2017;32:16-22.

4. Halstead SB, Suaya JA, Shepard DS. The burden of dengue infection. Lancet. 2007;369:1410-1. 
5. Dengue and severe dengue. World Health Organization. [Online]. Available from: http: //www.who. int/mediacentre/factsheets/fs117/en/.

6. Lin CC, Huang YH, Shu PY, Wu HS, Lin YS, Yeh TM, et al. Characteristic of dengue disease in Taiwan: 2002-2007. Am J Trop Med Hygiene. 2010;82: 731-9.

7. Yang CF, Hou JN, Chen TH, Chen WJ. Discriminable roles of Aedes aegypti and Aedes albopictus in establishment of dengue outbreaks in Taiwan. Acta Trop. 2014;130:17-23.

8. Wang SF, Chang K, Loh EW, Wang WH, Tseng SP, Lu PL, et al. Consecutive large dengue outbreaks in Taiwan in 2014-2015. Emerg Microbes Infect. 2016:5:e123.

9. Cao-Lormeau VM, Blake A, Mons S, Lastere S, Roche C, Vanhomwegen J, et al. Guillain-Barre syndrome outbreak associated with Zika virus infection in French Polynesia: a case-control study. Lancet. 2016;387:1531-9.

10. Rasmussen SA, Jamieson DJ, Honein MA, Petersen LR. Zika virus and birth defects--reviewing the evidence for causality. N Engl J Med. 2016; 374:1981-7.

11. Duong V, Lambrechts L, Paul RE, Ly S, Lay RS, Long KC, et al. Asymptomatic humans transmit denque virus to mosquitoes. Proc Natl Acad Sci U S A 2015;112:14688-93.

12. Ten Bosch QA, Clapham HE, Lambrechts L, Duong V, Buchy P, Althouse BM, et al. Contributions from the silent majority dominate dengue virus transmission. PLoS Pathog. 2018;14(5):e1006965.

13. Grange L, Simon-Loriere E, Sakuntabhai A, Gresh L, Paul R, Harris E. Epidemiological risk factors associated with high global frequency of inapparent dengue virus infections. Front Immunol. 2014;5:280.

14. Chen CJ, Lee PI, Chang SC, Huang YC, Chiu CH, Hsieh YC, et al. Seroprevalence and severity of 2009 pandemic influenza A H1N1 in Taiwan. PLoS One. 2011;6:e24440.

15. Peeling RW, Artsob H, Pelegrino JL, Buchy P, Cardosa MJ, Devi S, et al. Evaluation of diagnostic tests: dengue. Nat Rev Microbiol. 2010;8:S30-8.

16. Chien YW, Huang HM, Ho TC, Tsseng FC, Ko NY, Ko WC, et al. Seroepidemiology of dengue virus infection among adults during the ending phase of a severe dengue epidemic in southern Taiwan, 2015. BMC Infect Dis. 2019;19:338.

17. Dengue. Guidelines for Diagnosis, Treatment, Prevention and Control: New Edition. Geneva: World Health Organization; 2009. 4, LABORATORY DIAG NOSIS AND DIAGNOSTIC TESTS. Available from: https://www.ncbi.n/m.nih. gov/books/NBK143156/

18. Maldonado G, Greenland S. Simulation study of confounder-selection strategies. Am J Epidemiol. 1993;138:923-36.

19. Sultana N, Biswas SK, Sultan T, Ahmed S, Hossain Z, Chowdhury R. Seroprevalence of dengue fever in Chittagong, Bangladesh. Chatt Maa Shi Hosp Med Coll J. 2013;12:38-40.

20. Wilder-Smith A, Foo W, Earnest A, Sremulanathan S, Paton NI. Seroepidemiology of dengue in the adult population of Singapore. Tropical Med Int Health. 2004;9:305-8.

21. Ukey P, Bondade S, Paunipagar P, Powar R, Akulwar S. Study of seroprevalence of dengue fever in Central India. Indian J Commmunity Med. 2010;35:517-9.

22. Lo CL, Yip SP, Leung PH. Seroprevalence of dengue in the general population of Hong Kong. Tropical Med Int Health. 2013;18:1097-102.

23. Sa-Ngasang A, Anantapreecha S, A-Nuegoonpipat A, Chanama S, Wibulwattanakij S, Pattanakul K, et al. Specific IgM and IgG responses in primary and secondary dengue virus infections determined by enzymelinked immunosorbent assay. Epidemiol Infect. 2006;134:820-5.

24. Chien YW, Liu ZH, Tseng FC, Ho TC, Guo HR, Ko NY, et al. Prolonged persistence of IgM against dengue virus detected by commonly used commercial assays. BMC Infect Dis. 2018;18:156.

25. Chen WJ. Dengue outbreaks and the geographic distribution of dengue vectors in Taiwan: A 20-year epidemiological analysis. Biom J. 2018;41: 283-9.

26. Tsai JJ, Liu CK, Tsai WY, Liu LT, Tyson J, Tsai CY, et al. Seroprevalence of dengue in two districts of Kaohsiung city after the largest dengue outbreak in Taiwan since world war II. PLoS Negl Trop Dis. 2018;12: e0006879.

27. Research reports of Centers for Diseases Control, Department $f$ Health, Executive Yuan, 2007 (Reference No: DOH96-DC-2021) Seroepidemiologic study of dengue virus infection in southern Taiwan (Tainan city). available from: https://www.cdc.gov.tw/uploads/files/beda37f9-827c-497a-ab84-5b855 abe2d05.pdf
28. CDC. Taiwan National Infectious Disease Statistics System for dengue virus. Taiwan: Center for Disease Control; 2015. Available at: https://nidss.cdc.gov. tw/en/NIDSS_Diagram.aspx?dc=1\&dt=4\&disease $=061 \&$ position $=1$

\section{Publisher's Note}

Springer Nature remains neutral with regard to jurisdictional claims in published maps and institutional affiliations.

\section{Ready to submit your research? Choose BMC and benefit from:}

- fast, convenient online submission

- thorough peer review by experienced researchers in your field

- rapid publication on acceptance

- support for research data, including large and complex data types

- gold Open Access which fosters wider collaboration and increased citations

- maximum visibility for your research: over $100 \mathrm{M}$ website views per year

At $\mathrm{BMC}$, research is always in progress.

Learn more biomedcentral.com/submissions 